its development and it appeared in less intensity. This was repeated with similar results ; in this latter case crystals separated, which on being distilled with sodium carbonate gave no aldehyde reaction with the fuchsine-aldehyde reagent.

A portion of the oil was treated with dry ammonia gas for seven hours; there was no indication of any crystalline compound of aldehyde ammonia having been formed, and the oil gave the BechiMilliau test with even greater intensity.

\title{
TEST FOR SULPHUR COMPOUNDS.
}

In two cases only the black compound formed by the action of silver nitrate upon the fatty acid gave off hydrogen sulphide; this was recognized by its odor and blackening lead acetate paper. If, however, sulphur compounds were present in the fatty acid sufficient to blacken silver nitrate, it would seem as if other metallic salts should show characteristic colors. Salts of cadmium, lead, copper, and mercury were tried with negative results except that with the mercury salts reduction to metallic mercury took place.

In the repetition of Dupont's work both the aqueous distillate from the unheated oil and the oil itself gave the Bechi test. This was not the case with the oil which had been heated to $260^{\circ}$. In conclusion, in view of the fact that bodies of an aldehydic character could not be detected in the oil by either the fuchsine aldehyde reagent, by sodium bisulphite, or by ammonia, it would seem that it was not likely that they nere present. This would seem to be confirmed by Raikow's oxidation with permanganate and sulphuric acid. The evidence would seem to favor the presence of sulphur compounds.

\section{NEW BOOKS.}

STUDIES FROM THE CHEMICAL LABORATORY OF SHEFFIELD SCIENTIFIC Schoor. EDITED by Horace I. Weirs. Vol. I, xi 444 pp. Vol. II, ix 379 pp. New York: Charles Scribner's Sons. Igor. Price, $\$ 7.50$ per set.

In consequence of the recent bicentennial celebration of Yale University, there has been presented to the world "a series of volumes $* * *$ prepared $* * *$ and issued $* * *$ as a partial indication of the character of the studies in which the University teachers are engaged." Two volumes of this series, 
bearing the title given in the head lines, have been placed in the hands of the writer, who has read them with the greatest pleasure. The first volume contains a brief sketch of the Sheffield Laboratory, including a list of the publications (I3I) of the present officials of the department and their colaborers, followed by a series of papers in detail relating to general inorganic chemistry. Although the writer had studied these investigations as they appeared in print in the various journals, he can truthfully say that from the moment his attention was again attracted to the first lines of the first article which read "in the course of some experiments with caesium compounds, bromine was added to a concentrated solution of caesium chloride with an astonishing result," his interest in the remarkable results obtained in the preparation and study of double halides, by Professor Wells and his associates, was revived; indeed, this interest became so great that he read on and on, through one paper after the other, unconscious of his surroundings and the flight of time until the final paragraph was reached, when the book was closed with the conviction that it was overflowing with facts, bristling with suggestions, and deserving of the most careful study by all chemists, but especially those who fail to realize the immense work remaining to be done in the inorganic field. Five hundred double halides have been studied, more than a third of which have been made in the Yale laboratory. It is not very surprising then to read in the final chapter," "that the valency of a negative halide has no influence upon the types of double salts that it forms;" that " molecules of alkaline halides possess nearly the same combining power as negative halides; that double halides probably increase in ease of formation and variety from the iodides to the fluorides," and then "some indications of regularity have been observed in connection with these researches, but it must be admitted that the results have been negative as far as throwing light upon the structure of this class of compounds is concerned."

While the prevailing thought in Volume $I$ is "double halogen salts," we meet in the companion volume anilides, formyl derivatives, amidines, imido esters, thiazoles, urea amidines, acyclic benzoyl pseudoureas and thiosemicarbazidic esters-all attesting, by the skilful way in which they have been prepared and studied, the keen originating power, and the untiring energy and zeal of their various authors. Just a bit of the "double halide" spirit 
has found its way into several of these organic papers, for upon p. 34 will be noticed

$$
\begin{aligned}
& \left(\mathrm{C}_{6} \mathrm{H}_{4} \mathrm{NO}_{2} \cdot \mathrm{NH} \cdot \mathrm{CO} . \mathrm{CH}_{3}\right)_{2} \mathrm{HBr} \cdot \mathrm{Br}_{2}, \\
& \left(\mathrm{C}_{6} \mathrm{H}_{4} \mathrm{NO}_{2} \cdot \mathrm{NH} . \mathrm{CO} \cdot \mathrm{CH}_{3}\right)_{2} \mathrm{HBr} \cdot \mathrm{Br}_{4} \text {, etc., }
\end{aligned}
$$

which are perfectly analogous to the perhalides $\mathrm{CsBr} . \mathrm{Br}_{2}, \mathrm{CsI}_{4}$, and $\mathrm{NH}_{4} \mathrm{Br}$. $\mathrm{Br}_{2}$. This slight digression into the field that received such ample consideration in the first volume is most pardonable, nay, it is desirable and should be pursued farther whenever it is at all possible. Much more might be said, but as the character of the volumes is now in a measure indicated, the writer would conclude with the recommendation that the reader of these lines should study the papers for himself, feeling assured that when he has done so, he will concur with the writer in the opinion that these researches not only reflect great credit upon their authors and the university in which they were planned, but that they will be real incentives to the great body of young, enthusiastic chemists to go forward and do likewise.

EDGAR F. SMITH. 